\title{
An incremental stress-based constitutive modeling on anisotropic damaged materials
}

\author{
C.L. CHOW ${ }^{1}$, Y.J. LIU $^{2}$ and A. ASUNDI ${ }^{2}$ \\ ${ }^{1}$ Department of Mechanical Engineering, The University of Michigan-Dearborn, Dearborn, MI 48128-1491, USA \\ ${ }^{2}$ Department of Mechanical Engineering, University of Hong Kong, Pokfulam Road, Hong Kong
}

Received 15 November 1992; accepted in revised form 25 October 1993

\begin{abstract}
An 'incremental form' of anisotropic damage constitutive equation is proposed both for brittle and ductile materials. Based on the concept of irreversible thermodynamics that damage processes are history independent coupled with irreversible energy dissipation, two types of definition for damage representation are established, known as damage tensor $\mathbf{D}$ and damage strain tensor $\varepsilon^{d}$, to describe constitutive responses of damaged materials. A state variable coupled with damage and other observable state variables, i.e. $\varepsilon^{d}$, is formulated separately from other internal variables and defined as an equivalent damage variable. A constitutive relation due to damage is finally formulated by introducing 'damage flow potential function' employing the theory of irreducible integrity bases. A clear physical representation based on theoretical foundations and rigorous mathematical arguments of the conventional damage models defined in terms of 'damage effect tensor $\mathbf{M}(\mathbf{D})$ ' is also elucidated. Validity of the proposed model is verified by comparing with the formulations of conventional damage effect tensor. A plastic potential function coupled with damage is also introduced by employing the anisotropic plastic flow theory, so that the proposed damage model can be applied to characterize a wide range of damage problems of practical engineering interest.
\end{abstract}

\section{Introduction}

Experimental evidence has revealed that the phenomena of material degradation are induced by microdefects such as microcracks, microvoids and cavities, etc. (Murakami [1]). In addition, this material degradation even exhibits to some extent the changes of material macroscale characters, i.e. stiffness and hardness reduction or residual life decrease associated with irreversible thermodynamic processes. To rationally describe these behaviors and then to precisely predict the remainder life or strength of a material element is one of the most lively research areas in recent solid mechanics development. However, due to complex and random material microstructures, to characterize the material behavior precisely in microscale would require detailed knowledge of all microstructures in that material, thus rendering the problem too complex for engineering applications. One way to overcome this difficulty is the concept of damage mechanics (DM) inaugurated by Kachanov [2] and advocated subsequently by Lemaitre [3] and Lemaitre and Chaboche [4] etc. The essential idea and advantage of DM are to summarize the effects of microdefects in a material element with the changes of macroscale internal variables, called damage variables, based on the concept of a local volume average with statistical homogeneity by ignoring the microstructure details.

It is well accepted that the effects of damage on materials are manifested in changes in material stiffness or some equivalent parameters and these changes in macroscale can usually be measured by conventional standard experimentation using laboratory-size specimens. Different damage models have been formulated in the past based on the method known as the macro-mechanics approach due to Krajcinovic [5], Lemaitre [6], Murakami [7] and Chaboche [8-9] etc. Among them, a very important damage concept is the introduction of effective stress and effective stiffness, proposed initially by Lemaitre [3] and Sidoroff [10] and can be simply 
represented respectively as

$$
\tilde{\boldsymbol{\sigma}}=\mathbf{M}(\mathbf{D}): \boldsymbol{\sigma}
$$

and

$$
\widetilde{\mathbf{E}}(\mathbf{D})=[\mathbf{M}(\mathbf{D})]^{-1, T}: \mathbf{E}:[\mathbf{M}(\mathbf{D})]^{-1},
$$

where $\mathbf{M}(\mathbf{D})$ denotes damage effect tensor; $\tilde{\boldsymbol{\sigma}}$ and $\tilde{\mathbf{E}}$ are effective stress and effective stiffness, respectively. This type of damage concept has been successfully employed to solve a number of practical problems, including the assessment of life expectancy and the prediction of crack initiation by Kachanov [11] and Chow and Wang [12-15]. However the physical meaning of tensor $\mathbf{M}(\mathbf{D})$, especially for the component of $M_{i j}(i \neq j)$ when anisotropic damage problems are considered, has not been successfully interpreted physically or even fully understood. Moreover, according to later discussion in Section 2, this damage definition has in reality imposed a very strong restriction on the damage problems, and thus, has limited its applicability, e.g. the uni-axial crack damage problems. In order to surmount the restrictions imposed on the conventional damage model, a new alternative damage characterization is proposed in Sections 2 and 3. The incremental form of damage-stress relations based on the new damage characterization is derived such that the damage model can be discretized to perform finite element analysis for the solution of not only proportional but also non-proportional loading problems. Our effort is also focused on providing a physical representation of damage as an infinitesimal deforming process in Section 3. Several example cases are examined and then compared with those derived from the conventional damage models to demonstrate the validity of the proposed concept in Section 4. In Section 5, a coupled plastic potential function with damage variables is also presented and discussed.

\section{On the damage representation}

Broadly speaking, there are two major types of damage model, each developed based on its different physical interpretations of the mechanism of damage. One such damage model monitors state variable changes at macro-scale level by means of damage variables and may be known as a macro-damage model. Its essential hypothesis is that damage can be viewed as a macroscopic state variable by ignoring the micro-detail in the material element. This concept was first introduced by Kachanov in 1958 in the analysis of creep and fatigue problems [2], and later extended by Lemaitre and Chaboche $[3,4]$ who proposed the concept of 'effective strain' defined for isotropic damage as

$$
\tilde{\boldsymbol{\sigma}}=\frac{\sigma}{(1-D)}
$$

where $D$ is a scalar damage variable and $\tilde{\sigma}$ represents effective stress tensor. Since many damaged materials often exhibit anisotropic characters even if their initial property is isotropic, this expression of effective stress has been extended to a generalized tensorial form by Sidoroff 
[10] based on the concept of elastic energy equivalence. When the principle axes $\left(x_{1}, x_{2}, x_{3}\right)$ of stress coincide with the damage variable principle directions, (2.1) becomes

$$
\tilde{\boldsymbol{\sigma}}=\mathbf{M}(\mathbf{D}): \boldsymbol{\sigma},
$$

where the Voigt notation is adopted

$$
\sigma=\left(\sigma_{1}, \sigma_{2}, \sigma_{3}\right) ; \quad \tilde{\boldsymbol{\sigma}}=\left(\tilde{\sigma}_{1}, \tilde{\sigma}_{2}, \tilde{\sigma}_{3}\right)
$$

and $\mathbf{M}(\mathbf{D})$ defined as 'damage effect tensor (DET)' is expressed as

$$
\mathbf{M}(\mathbf{D})=\left[\begin{array}{ccc}
\frac{1}{1-D_{1}} & & \\
& \frac{1}{1-D_{2}} & \frac{1}{1-D_{3}}
\end{array}\right] .
$$

Recently, this formulation of $\mathbf{M}(\mathbf{D})$ has been further developed in a number of generalized forms $[1,7,12-14]$. The validity of the DET formulations due to Chow and his co-workers has been checked experimentally as well as its applicability to solve a number of crack initial and propagation problems [12-16].

Based on the assumption of strain energy equivalence, the effective compliance can be represented according to the definition of $\mathbf{M}(\mathbf{D})$ as $[10,13]$

$$
\widetilde{S}(\mathbf{D})=\mathbf{M}^{\mathrm{T}}(\mathbf{D}): \mathbf{S}: \mathbf{M}(\mathbf{D}) \text {. }
$$

From the point of material science, the material deterioration in the process of deformation or/and damage initiation, growth and coalescence is entirely determined by material microstructural rearrangements as a form of thermodynamic irreversible process. Thus, the exact analytical expression of damaged material stiffness $\widetilde{\mathbf{S}}(\mathbf{D})$ as well as the damage effect tensor $\mathbf{M}(\mathbf{D})$ should be unique in the characterization of a particular material and its associated damage entities. As mentioned before, there are various formulations of DET for the characterization of a material and its damage entities. This unfortunate state of affairs is largely attributed to the arbitrariness of each formulation without sound fundamental and theoretical arguments. This arbitrariness results primarly from the lack of physical interpretation and representation of DET, especially for the components of $M_{i j}(i \neq j)$. On the other hand, the damage variables defined based on the effective stress and/or the effective compliance, are imposed with a particular restriction which, as indicated in (2.12) and (2.5) dictates the formulation of the damage effects on the material properties. For example, consider a particular damage entity for which the component $D_{2}$ vanishes, i.e. a uni-axial damage system developed in continuous fiber reinforced laminated materials due to matrix-cracking. Then according to (2.5) for plane stress problems, the effective Young's modulus is

$$
\tilde{\mathbf{E}}_{1}=\frac{\mathbf{E}_{1}}{\left(1-\mathbf{D}_{1}\right)^{2}}
$$


and effective Poisson's ratio is

$$
\tilde{\mu}_{12}=\frac{\mu_{12}}{\left(1-\mathbf{D}_{1}\right)}
$$

From simple tensile test for laminate materials, it has been observed that the above relations violate experimental results, i.e. reduction of Poisson's ratio is much larger than that of Young's modulus rather than lower as indicated in (2.6) and (2.7) [17].

Another major type of damage model is advocated by Vakulenko and Kachanov [18], Krajcinovic and Fonseka [19], Talreja [20] and Allen [21] and directly formulates the damage variables based on micro-discontinuity of some state variables such as micro-displacement or other micro-structural irreversible changes. We may identify this type of damage model as a micro- or mesco-scale damage model. Consider a representative volume $V$ containing $k$ damage entities such as cracks or crack-like defects. The damage variable is generally defined by statistical average of the volume as proposed in [18]

$$
D_{i j}=\frac{1}{V} \sum_{k} \int_{s(k)} b_{i}^{k} \cdot n_{j}^{k} \cdot \mathrm{ds}(k)
$$

where $n_{i}$ is unit vector outward normal to the surface of a single representative entity; $b_{j}$ is a properly selected micro-discontinuous value, e.g. the micro-displacement of a point on $s(k)$ due to damage entity according to Allen [21] and the extent and direction of some 'micro-influence' proposed by Talreja [20], while $s(k)$ denotes the surface of the $k$ th crack in the material volume in question. It is obvious that the advantage of this type of definition of damage variable is its clear physical representation of material damage. However in many published works some authors argued that the damage variable so defined is in reality associated with observable displacements of a material body, which does not consequently satisfy the basic assumptions of damage as an internal variable. Specifically, Murakami [1] pointed out that: 'although a plane crack $b_{i}=0$ (see (2.8)) surely induces a certain material damage, it does not make any contribution to $D_{i j}$ expressed in (2.8)'. Furthermore, the damage variable defined in terms of micro-displacement does not remain constant in reversible processes, say when a material element after a load application is completely unloaded, due to the inherent deficiency in definition of $b_{i}$.

Consider permanent micro-structure changes of a damaged material due to micro-cracks, micro-voids and other 'observable geometric shaped defects'. A second order tensoral representation such as (2.8), expressed by $\mathbf{D}$, can be found in many works. Similarly, we propose to modify the equation due to its lack of physical representation mentioned earlier, with the following general definition of damage tensor $\mathbf{D}$ as

$$
\mathbf{D}=\frac{1}{V} \sum_{K} \int_{S(K)} \mathbf{D}^{k} \cdot \mathrm{d} S(k)
$$

where

$$
\mathbf{D}^{k}=\mathbf{D}^{k}\left(D^{k}, g^{k}, \alpha^{k}(n), \ldots, \mathbf{V}^{k}, \mathbf{b}^{k}, \mathbf{V}_{\otimes}^{\mathrm{k}} \mathbf{b}^{k}, \mathbf{V}_{\otimes}^{\mathrm{k}} \mathbf{V}^{k}, \mathbf{b}_{\otimes}^{\mathrm{k}} \mathbf{b}^{k}, \ldots\right)
$$


where $\mathbf{b}^{k}$ is defined as the residual micro-displacement vector at a point on $s(k)$ between the two relative sides of the $s(k)$ th crack when the damaged material is completely unloaded; $V^{k}$ represents the components of matrix crack area vector of $s(k) ; D^{k}$ and $g^{k}$ are the density of matrix cracks and the coefficient of crack geometry, respectively, while $\alpha^{k}(n)$ are the parameters due to the material secondary damage effects, such as the interaction between cracks, stress concentration, etc.

It is well-accepted that a damaged material often exhibits varying material symmetric properties, i.e. isotropic, transverse isotropic or orthotropic symmetry. In addition, these symmetric properties of damaged material are sufficiently determined by the damage variable coupled with its virgin properties of material. For ease of illustration, we confine our attention, similar to other conventional studies, on the orthotropic damaged materials, although, rigorously speaking, damaged materials often behave anisotropically. Furthermore, virgin materials considered in this paper are assumed as isotropic ones. Since the damage variable defined as an internal state variable which can completely characterize the irreversible material structure changes, it is consequently reasonable to assume that the damage tensor has the same symmetric property as the damaged material, i.e. orthotropic symmetry under the preferred directional coordinate system. However, it should be noted that the preferred directions of a virgin orthotropic material do not generally coincide with the principle axes of damage variable, so the eventual preferred directions of a damaged material are determined by both the virgin and damage variable principle axes rather than by damage alone.

Mathematically, the symmetry of damage variable may be expressed in terms of corresponding orthogonal transformations in its material preferred coordinates system $X\left(x_{1}, x_{2}, x_{3}\right)$. Without loss of generality, if the three mutually perpendicular unit vectors, $\left(e_{1}, e_{2}, e_{3}\right)$ are the preferred directions of the damaged material, the associated transformations, $\mathbf{R}_{1}, \mathbf{R}_{\mathbf{2}}$ and $\mathbf{R}_{\mathbf{3}}$ in $\left(e_{1}, e_{2}, e_{3}\right)$ coordinate system for its orthotropic symmetry, e.g. Rhombic-dipyramidal, may be presented as

$$
\begin{aligned}
& \mathbf{R}_{1}=\operatorname{diag}(1,-1,-1) ; \quad \mathbf{R}_{2}=\operatorname{diag}(-1,1,-1) ; \\
& \mathbf{R}_{\mathbf{3}}=\operatorname{diag}(-1,-1,1) .
\end{aligned}
$$

Substitute the above into (2.10) and take into account a second order approximation. The individual first order terms of $\mathbf{b}^{k}$ and $\mathbf{V}^{k}$ vanish due to the orthotropic symmetry. In addition, the coefficients $a_{i j}^{k}, b_{i j}^{k}$ and $c_{i j}^{k}$, which are damage coefficients dependent on $D^{k}, g^{k}$ and $\alpha^{k}(n)$, are also reduced to zero for $i \neq j$. Therefore, with the initial condition that $\mathbf{D}=0$, when $\mathbf{V}=0$, the damage variable can be approximated by

$$
D_{i j}^{k}=a_{i j}^{k} \cdot V_{1}^{k} \cdot V_{j}^{k} \cdot \delta_{i j}+b_{i j}^{k} \cdot V_{l}^{k} \cdot b_{j}^{k} \cdot \delta_{i j}, \quad \text { (no summation on } i \text { and } j \text { ). }
$$

(2.12) may be alternatively expressed as

$$
\mathbf{D}=\left[\begin{array}{ccc}
D_{11} & D_{12} & D_{13} \\
& D_{22} & D_{23} \\
s & & D_{33}
\end{array}\right] .
$$


Similarly, for isotropic damage problems, two additional transformations $\mathbf{R}_{\boldsymbol{\theta}}$ and $\mathbf{R}_{\phi}$ are satisfied, where $\mathbf{R}_{\theta}$ and $\mathbf{R}_{\phi}$ represent a rotation about $x_{1}$ and $x_{3}$, respectively. This results in $D_{11}^{k}=D_{22}^{k}=D_{33}^{k}=D$ in (2.14), and damage $\mathbf{D}$ is thus reduced to

$$
\mathbf{D}=D \cdot \operatorname{diag}(1,1,1)
$$

which is equivalent to a scalar damage variable for isotropic damage as in the conventional damage models.

On the other hand, not all the material deterioration can be summarized by the proposed damage tensor, such as internal friction, dislocation movement etc. In fact, when the damage tensor $\mathbf{D}$ vanishes, the material internal friction may also lead to the reduction and degradation of material macro-properties, e.g. stiffness, especially for fatigue problems. Furthermore, these damage entities generally behave without any significantly 'preferred' directions, or displaying isotropic property. A brief review from the existing literature reveals that the internal variables, i.e. $\omega, \alpha$, etc., are associated with these types of damage. In this paper, we also define them as damage variables and name $\omega$ as 'overall damage' to characterize the 'isotropic' damage entities and its coupled effects with damage tensor $\mathbf{D}$.

\section{Brittle anisotropic damage}

The constitutive responses of damaged material are related to irreversible thermodynamic processes involving some material macro-property changes, such as stiffness reduction or/and energy dissipation. According to the assumptions (ii) and (iii) mentioned above, such irreversible thermodynamic processes can be described by a sequence of constrained equilibrium states corresponding to the instantaneous values of internal variables (damage variable), which is history and process independent.

From the above assumptions, we may decompose the description of material deformation. Consider an infinitesimal deformation process in a material element coupled with damage entity. A reference configuration $X_{t}$ of a damaged material body at time $t$ and represented as $X_{t}(\sigma, \varepsilon, \mathrm{D})$, see Fig. 1, where D represents the damage variable proposed in Section 2 . The deformation to be examined is defined as a state change from $X_{t}$ to $X_{t+\mathrm{d} t}(\sigma+d \sigma, \varepsilon+\mathrm{d} \varepsilon$, $\mathbf{D}+d \mathbf{D})$. Suppose a reference middle-state is $X_{t}^{\prime}(\boldsymbol{\sigma}+d \sigma, \boldsymbol{\varepsilon}+d \hat{\varepsilon}, \mathbf{D})$, which suffers no damage change from the process $X_{t}$ to $X_{t}^{\prime}$ and thus no energy dissipation is involved. As illustrated in Fig. 1, the displacement fields according to the deforming processes may be represented separately as

$$
\begin{aligned}
& X_{t} \text { to } X_{t+\mathrm{d} t}: d u=\boldsymbol{O P}-\boldsymbol{O P}, \\
& X_{t} \text { to } X_{t}^{\prime}: d \hat{u}=\boldsymbol{O P}-\boldsymbol{O P}
\end{aligned}
$$

and

$$
X_{t}^{\prime} \text { to } X_{t+d t}: d u^{d}=\boldsymbol{O P}-\boldsymbol{O P}
$$




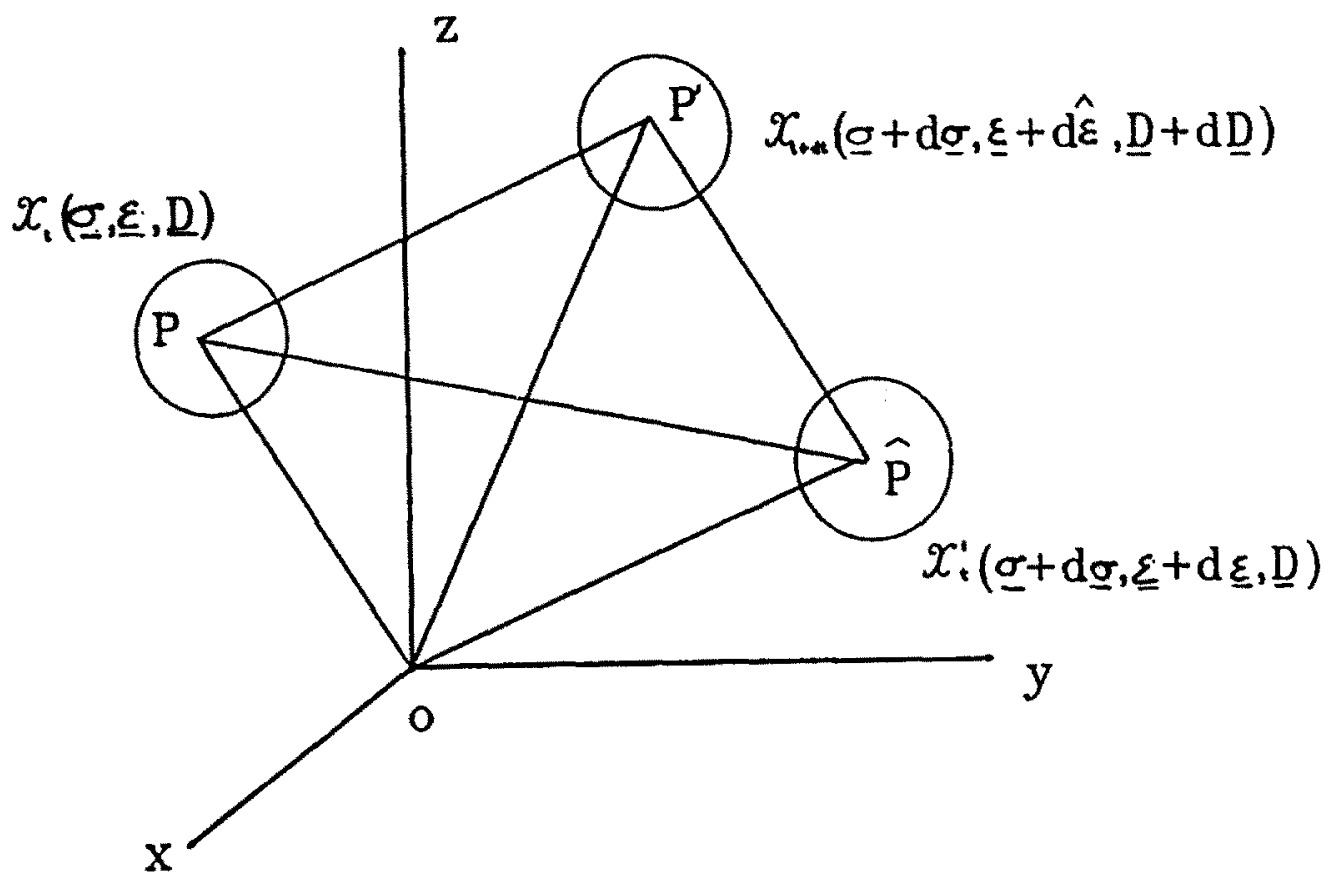

Fig. 1. Illustration of deformation processes due to damage.

Therefore, from the deformation theory, we have

$$
d \varepsilon=d \hat{\varepsilon}+d \varepsilon^{d}
$$

where $d \boldsymbol{\varepsilon}, d \hat{\varepsilon}$ and $d \varepsilon^{d}$ are strain tensors determined by $d \boldsymbol{u}, d \hat{\boldsymbol{u}}$ and $d \boldsymbol{u}^{\mathrm{d}}$, respectively. Without loss of generality, for brittle materials, $d \varepsilon$ is an increment of current elastic strain tensor; $d \hat{\varepsilon}$ is defined as an increment of partial elastic strain tensor at constant damage state $\mathbf{D}$ and $d \hat{\varepsilon}^{d}$, an increment of damage strain tensor. The corresponding displacement fields $d \boldsymbol{u}$, $d \hat{\boldsymbol{u}}$ and $d \boldsymbol{u}^{\mathrm{d}}$ are known as increment of current, partial and damage displacement fields, respectively. It should be emphasized that the effects of damage on the properties of deformed material may thus be entirely characterized by $\boldsymbol{\varepsilon}^{\mathrm{d}}$.

From (3.4) and without loss of generality, the increment of stress may be readily given as

$$
d \boldsymbol{\sigma}=\mathbf{E}(\mathbf{D}, \omega): d \varepsilon-\mathbf{E}(\mathbf{D}, \omega): d \varepsilon^{d}
$$

where $\mathbf{E}(\mathbf{D}, \omega)$ is the equivalent stiffness with damage $\mathbf{D}$ and the overall damage $\mathbf{u}$. A clear geometrical explanation of (3.5) may be found in Fig. 2, where $A C=d \varepsilon^{d}, H G=d \sigma$ and $A B=d \varepsilon$.

From the point of continuum irreversible thermodynamics, the law of physical damage process for a material undergoing progressive deterioration, i.e. micro-cracking, is generally known as the conservation of energy (first law) and the production of entropy (second law). With our attention confined to the purely mechanical deformation processes with quasi-static damage growth, the first law of thermodynamics requires that

$$
d W=d W^{e}+d W^{d},
$$




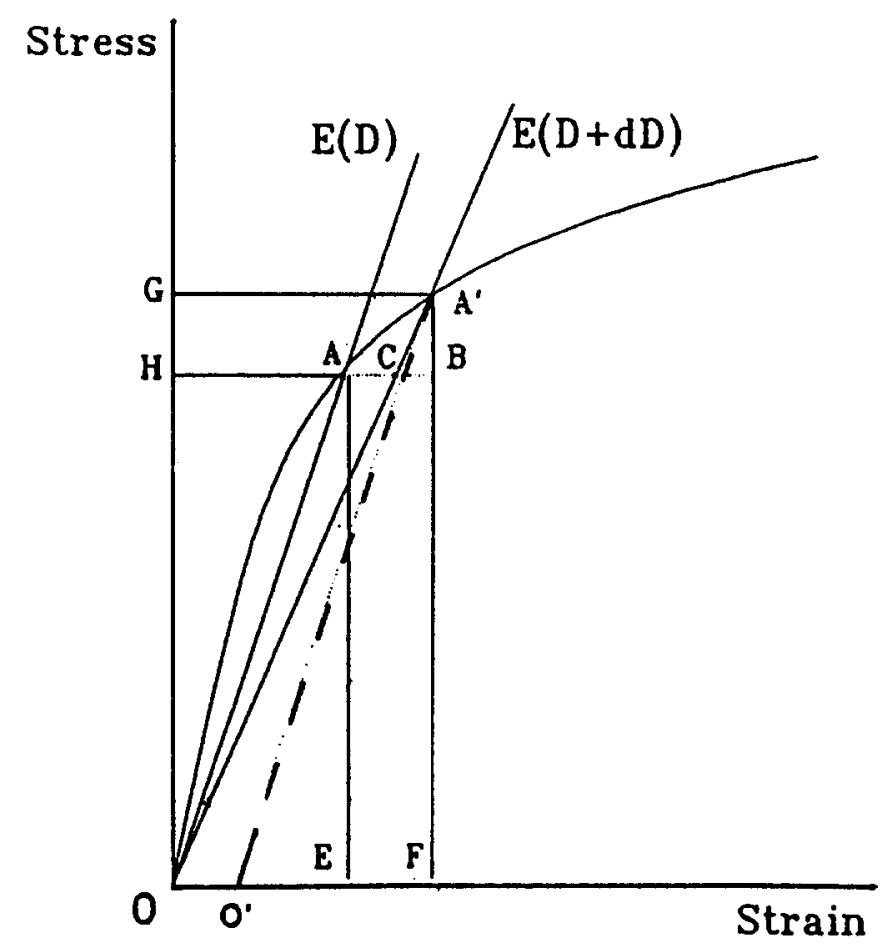

Fig. 2. Illustration of deformation from $X_{t}$ to $X_{1+\mathrm{d} t}^{\prime}$ for damaged materials.

where $d W=\sigma: d \varepsilon$ represents the increment of work of applied load, $d W^{e}$ and $d W^{d}$ denote the increment of material elastic strain energy and the dissipative work done on damage nucleation and propagation, respectively. Consider the elastic energy graphically illustrated in Fig. 2 as the area of $O A E$

$$
W^{e}=\operatorname{area}(O A E)=\frac{1}{2} \sigma: \varepsilon
$$

Then the increment of elastic energy $d W^{e}$ is derived as

$$
\begin{aligned}
d W^{\boldsymbol{e}} & =\operatorname{area}\left(O A^{\prime} O^{\prime}\right)=\frac{1}{2}(d \boldsymbol{\sigma}: \boldsymbol{\varepsilon}+\boldsymbol{\sigma}: d \boldsymbol{\varepsilon}) \\
& =\boldsymbol{\varepsilon}: \mathbf{E}(\mathbf{D}, \omega): d \boldsymbol{\varepsilon}-\frac{1}{2} \boldsymbol{\varepsilon}: \mathbf{E}(\mathbf{D}, \omega): d \boldsymbol{\varepsilon}^{d}
\end{aligned}
$$

where $d \boldsymbol{\sigma}$ is given by (3.5)

Conventionally, the form of free energy function $\Phi$ is often formulated in conjunction with the material elastic energy. However, an exact expression of $\Phi$ may sometimes be very difficult to obtain analytically. Instead of directly defining the free-energy function $\Phi$, we introduce an alternative definition in incremental form. From (3.8), an incremental form of free-energy potential $\Phi$ is considered as

$$
\begin{aligned}
& d \Phi\left(\boldsymbol{\varepsilon}, \boldsymbol{\varepsilon}^{d}, \mathbf{D}, \omega, t\right)=d W^{\boldsymbol{e}}+d\left(\Phi_{1}(\omega)\right) \\
& \quad=\frac{\partial \Phi}{\partial \boldsymbol{\varepsilon}}: d \boldsymbol{\varepsilon}+\frac{\partial \Phi}{\partial \boldsymbol{\varepsilon}^{\mathrm{d}}}: d \boldsymbol{\varepsilon}^{d}+d\left(\Phi_{1}(\omega)\right)=\boldsymbol{\sigma}: d \boldsymbol{\varepsilon}-\boldsymbol{\sigma}^{d}: d \varepsilon^{d}+d\left(\Phi_{1}(\omega)\right) \\
& \quad=\varepsilon: \mathbf{E}(\mathbf{D}, \omega): d \boldsymbol{\varepsilon}-\frac{1}{2} \varepsilon: \mathbf{E}(\mathbf{D}, \omega): d \varepsilon^{d}+d\left(\Phi_{1}(\omega)\right),
\end{aligned}
$$


where

$$
\begin{aligned}
& \boldsymbol{\sigma}=\frac{\partial \Phi}{\partial \boldsymbol{\varepsilon}}=\mathbf{E}(\mathbf{D}, \omega): \boldsymbol{\varepsilon} ; \quad \boldsymbol{\sigma}^{d}=-\frac{\partial \Phi}{\partial \boldsymbol{\varepsilon}^{d}}=\frac{1}{2} \mathbf{E}(\mathbf{D}, \omega): \boldsymbol{\varepsilon} \\
& B_{1}=-\frac{\partial \Phi}{\partial \omega}=-\Phi_{1}^{\prime}(\omega) .
\end{aligned}
$$

Note that $\Phi$ is a hidden function of damage $\mathbf{D}$ as shown in (3.8). From (3.6), (3.8) and (3.9), the second law of thermodynamics takes the form

$$
\boldsymbol{\sigma}^{d}: d \varepsilon^{d}+B_{1} \cdot d \omega \geqslant 0
$$

which indicates that $\sigma^{d}$ is the associated variable of $\varepsilon^{d}$.

On the other hand, based on the conventional argument of elastic energy as well as the free energy function for damaged materials, $\Phi$ can also be formulated in terms of associated elastic strain energy. However, the effective stiffness $\tilde{\mathbf{E}}$ is often assumed to be determined only by $\mathbf{D}$ as adopted in the conventional models. Accordingly, $\Phi$ may be given by

$$
\Phi=W^{e}+\Phi_{2}(\omega)=\frac{1}{2} \varepsilon: \mathbf{E}(\mathbf{D}, \omega): \varepsilon+\Phi_{2}(\omega)
$$

Then from (3.12)

$$
d \Phi=\boldsymbol{\sigma}: d \boldsymbol{\varepsilon}-\mathbf{Y}: d \mathbf{D}-\boldsymbol{B} \cdot d \omega
$$

where $\mathbf{Y}$ signifies the associated force of damage variable $\mathbf{D}$ and is also known as the damage strain energy release rate.

$$
\mathbf{Y}=-\frac{\partial \Phi}{\partial \mathbf{D}}=-\frac{1}{2} \boldsymbol{\varepsilon}: \frac{\partial \mathbf{E}(\mathbf{D}, \omega)}{\partial \mathbf{D}}: \boldsymbol{\varepsilon}
$$

and $B$ is completely expressed as the associated force of $\omega$

$$
B=-\frac{\partial \Phi}{\partial \omega}=-\frac{1}{2} \varepsilon: \frac{\partial \mathbf{E}(\mathbf{D}, \omega)}{\partial \omega}: \varepsilon-\Phi_{2}^{\prime}(\omega)
$$

From (3.13) and (3.9), the Clausius-Duhem inequality becomes

$$
\mathbf{Y}: d \mathbf{D}+B \cdot d \omega=\boldsymbol{\sigma}^{d}: d \varepsilon^{d}+B_{1} \cdot d \omega \geqslant 0
$$

From the irreversible thermodynamics, a state variable is defined as an internal variable only if it is history independent. $\varepsilon^{d}$, as shown in (3.4), is however associated with material deformation processes, and is thus a history-dependent variable coupled with the effects of damage $\mathbf{D}$. In order to distinguish $\boldsymbol{\varepsilon}^{d}$ from the concept of internal variable, we may name the history-dependent state variable, which can to some extent, characterize the damage effects on the macro-properties of 
materials, as an equivalent damage variable. The implication of this concept as shown in (3.16) is that the dissipative energy due to damage can entirely be expressed with the proposed equivalent damage variable $\varepsilon^{d}$, such that material degradation may be formulated in terms of $\varepsilon^{d}$ instead of directly by $\mathbf{D}$. The relation between $\varepsilon^{d}$ and damage $\mathbf{D}$ will be discussed in the next section.

\section{Damage evolution}

\subsection{Damage evolution law}

From the irreversible process of thermomechanics, the inequality of (3.11) is associated with a properly defined flow potential function $\hat{F}_{d}\left(\sigma^{d}, \mathbf{D}\right)[16]$. Without loss of generality, we consider

$$
F_{d}=\hat{F}_{d}\left(\sigma^{d}, \mathbf{D}\right)-F_{2}\left(B_{1}\right)
$$

Then with the assumption of convex and normality, we obtain

$$
\begin{aligned}
& d \varepsilon^{d}=d \lambda \cdot \frac{\partial F_{d}}{\partial \sigma^{d}} \\
& d \omega=d \lambda \cdot \frac{\partial F_{d}}{\partial\left(-B_{1}\right)}=d \lambda \cdot F_{2}^{\prime}\left(B_{1}\right)
\end{aligned}
$$

where $d \lambda$ is a non-negative scalar parameter. As the condition of damage flow is postulated to satisfy $d F_{d}\left(\sigma^{d}, \mathbf{D}\right)=0$, we may deduce

$$
\begin{array}{ll}
d \lambda=0 & F_{d}<0 \text { or } F_{d}=0 \text { and } \\
& \frac{\partial \hat{F}_{d}}{\partial \boldsymbol{\sigma}^{d}}: d \sigma^{d}+\frac{\partial \hat{F}_{d}}{\partial \mathbf{D}}: d \mathbf{D}<=0 \\
d \lambda=\frac{\frac{\partial \hat{F}_{d}}{\partial \boldsymbol{\sigma}^{d}}: d \sigma^{\mathbf{d}}+\frac{\partial F_{d}}{\partial \mathbf{D}}: d \mathbf{D}}{\left(F_{2}^{\prime}\left(B_{1}\right)\right)^{2} \cdot B^{\prime}(\omega)} & \left.\begin{array}{l}
F_{d}>=0 \text { and } \\
\frac{\partial \hat{F}_{d}}{\partial \boldsymbol{\sigma}^{d}}: d \sigma^{d}+\frac{\partial \hat{F}_{d}}{\partial \mathbf{D}}: d \mathbf{D}>0
\end{array}\right\}
\end{array}
$$

Similar definition of $d \lambda$ can also be found in the open literature such as [22], which referred to $d \lambda$ as Lagrange multiplier.

Consider a scalar function $\hat{F}_{d}$ determined by damage $\mathbf{D}$ jointly with $\sigma^{d}$. The general form of $\hat{F}_{d}$ may be expressed entirely by the irreducible integrity bases using the invariant theory. The associated irreducible integrity bases relating to the orthotropic damaged materials in question have been given by Spencer [23] and Talreja [24] under the orthogonal group of coordinate transformations, see Appendix I. Take a quadratic approach to $\hat{F}_{d}$ with $\sigma_{i j}^{d}$ and first order with damage tensor D. $\hat{F}_{d}$ may be in general represented as (using the Voigt notation and also see Appendix I (A1.2))

$$
\begin{aligned}
& \hat{F}_{d}=\hat{F}_{d}\left(I_{k}\right), \quad(k=1,2,-18) \\
& =J_{k}^{(1)}\left(D_{k 1}\right) \cdot \sigma_{k}^{d}+J^{(2)}\left(D_{k 1}\right)+0.5 \cdot J_{i j}\left(D_{k 1}\right) \cdot \sigma_{i}^{d} \cdot \sigma_{j}^{d}, \quad(k, l=1-3, \text { and } i, j=1-6),
\end{aligned}
$$


where $J_{i}^{(1)}\left(D_{k l}\right), J^{(2)}\left(D_{k l}\right)$ and $J_{i j}\left(D_{k l}\right)$ are dependent upon the damage variable D. Suppose $d \varepsilon^{d}$ vanishes in un-deformed state (or/and un-loading or/and compressing processes) for which $\sigma^{d}=0$. Then, according to (4.2) and (4.4), we have

$$
J_{k}^{(1)}\left(D_{k l}\right)=0 ; \quad J^{(2)}\left(D_{l k}\right)=0
$$

In addition, for cracking or crack-like damage entities, it is reasonable to assume that the effects of damage components along $x_{3}$-direction on the elastic characters in the plan $x_{1}-O-x_{2}$ normal to $x_{3}$-direction are negligible (see [9]). Then, the components of $J_{i j}\left(D_{k l}\right)$ may be readily obtained

$$
\begin{array}{ll}
J_{11}=a_{1}+a_{2} D_{11} ; & J_{22}=a_{3}+a_{4} D_{22} ; \\
J_{33}=a_{5}+a_{6} D_{33} ; & J_{12}=b_{4}+b_{5} D_{11}+b_{6} D_{22} ; \\
J_{23}=b_{1}+b_{2} D_{22}+b_{3} D_{33} ; & J_{13}=b_{7}+b_{8} D_{11}+b_{9} D_{33} ; \\
J_{44}=c_{1}+c_{2} D_{22}+c_{3} D_{33} ; & J_{55}=c_{4}+c_{5} D_{11}+c_{6} D_{33} \\
J_{66}=c_{7}+c_{8} D_{11}+c_{9} D_{22} ; & J_{45}=d_{1}+d_{2} D_{12} ; \\
J_{56}=d_{3}+d_{4} D_{23} ; \quad J_{46}=d_{5}+d_{6} D_{13} ; \\
J_{14}=e_{1}+e_{2} D_{23}=J_{24}=J_{34} \\
J_{15}=e_{3}+e_{4} D_{13}=J_{25}=J_{35} \\
J_{16}=e_{5}+e_{6} D_{23}=J_{26}=J_{36}
\end{array}
$$

and the others are zero. In addition,

$$
J_{i j}=J_{j i} \quad(\mathrm{i}, \mathrm{j}=1,2-6)
$$

where the coefficients $a_{i}, b_{j}, c_{j}$ and $d_{i}$ in (4.6) are material constants and may be measured experimentally.

Substitute (4.4) and (4.5) into (4.2)

$$
\begin{aligned}
& d \varepsilon^{d}=d \lambda \cdot \mathbf{J}(\mathbf{D}): \sigma^{d}=d \lambda \cdot \mathbf{J}(\mathbf{D}): \mathbf{E}(\mathbf{D}): \varepsilon, \\
& d \omega=d \lambda \cdot F_{2}^{\prime}\left(B_{1}\right)
\end{aligned}
$$

Combining (4.1), (4.2), and (4.8), we obtain

$$
d \varepsilon^{d, T}: \mathbf{J}^{-1}: d \varepsilon^{d}=(d \lambda)^{2} \cdot \sigma^{d, T}: \mathbf{J}: \sigma^{d}=\left[d \lambda \cdot F_{2}^{\prime}\left(B_{1}\right)\right]^{2} \frac{2 F_{2}\left(B_{1}\right)}{\left[F_{2}^{\prime}\left(B_{1}\right)\right]^{2}}
$$

Without loss of generality, choose $F_{2}\left(B_{1}\right)$ as

$$
F_{2}\left(B_{1}\right)=\frac{1}{2} \cdot B_{1}^{2}
$$

hence $B_{1}$ may be given

$$
B_{1}=B_{0}+\nu \cdot \omega^{k}
$$


$B_{0}$ is the initial damage hardening threshold $(\omega=0)$ and $v$ and $k$ are material constants to be determined experimentally. Therefore, substituting (4.9) into (4.10), $d \omega$ is derived as

$$
d \omega=\sqrt{d \varepsilon^{d, T}: \mathbf{J}^{-1}(\mathbf{D}): d \varepsilon^{d}}
$$

where $\mathbf{J}^{-1}(\mathbf{D})$ is the generalized inverse of tensor $\mathbf{J}(\mathbf{D})$.

Assume that the flow of damage evolution satisfies the normality relation with its associated force, then the evolution law of damage may be expressed in a generalized form as

$$
d \mathbf{D}=d \lambda \cdot \mathbf{G}(\mathbf{Y}, \mathbf{D}, \omega)
$$

The exact analytical expression of the tensorial function $\mathbf{G}(\mathbf{Y}, \mathbf{D}, \omega)$ should be made based on experiments and/or the detailed knowledge of physical properties of the microdefects.

To characterize the damage criterion in the sense of 'stress' increase coupled with damage such as (4.1) is known as a stress-based model which postulates that damage initiation and growth are developed only when $F_{d} \geqslant 0$. Similar definitions for $F_{d}$ have also been proposed in other works (e.g. [25]), one of which is called an energy-based model and argues that the damage criterion should more reasonably be formulated with the damage energy release rate or the damage conjugate force, as defined in this paper as $\mathbf{Y}$. In fact, according to the above discussion, the difference between the two models can be identified from examining (3.16) which signifies that their dissipative energies due to damage are the same. However, a major difference between the stress-based and the energy-based models is their respective definition of the equivalent stiffness due to damage. For the energy-based model, the equivalent stiffness is directly formulated in terms of $\mathbf{E}(\mathbf{D}, \omega)$ as shown in (3.12), while the stress-based model with the damage potential function, in terms of $\mathbf{J}(\mathbf{D})$. The validity of the two models will be examined by comparing with other conventional models available in the literature in the next section.

\subsection{The equivalent stiffness and compliance}

Consider the following stress-strain relations (unless otherwise specified, we simply express $\mathbf{E}(\mathbf{D}, \omega)$ as $\mathbf{E}(\mathbf{D})$ in subsequent discussions) as

$$
\begin{aligned}
& \sigma=\mathbf{E}(\mathbf{D}): \varepsilon \\
& \sigma+d \boldsymbol{\varepsilon}=\mathbf{E}(\mathbf{D}+d \mathbf{D}):(\varepsilon+d \varepsilon)
\end{aligned}
$$

Substitute (3.5) and (4.8) into (4.12) with $\mathbf{E}(\mathbf{D}+d \mathbf{D})$ approximated by

$$
\mathbf{E}(\mathbf{D}+d \mathbf{D})=\mathbf{E}(\mathbf{D})+d \mathbf{E}(\mathbf{D})
$$

for which higher order infinitesimal terms are neglected, the increment of damaged material equivalent stiffness is derived as

$$
\mathbf{E}(\mathbf{D}+d \mathbf{D})=\mathbf{E}(\mathbf{D})-\frac{1}{2} d \lambda \cdot \mathbf{E}(\mathbf{D}): \mathbf{J}(\mathbf{D}): \mathbf{E}(\mathbf{D})
$$


Similarly,

$$
\begin{aligned}
& \boldsymbol{\varepsilon}+d \boldsymbol{\varepsilon}=\mathbf{S}(\mathbf{D}+d \mathbf{D}):(\sigma+d \sigma), \\
& \boldsymbol{\varepsilon}=\mathbf{S}(\mathbf{D}): \boldsymbol{\sigma},
\end{aligned}
$$

where $\mathbf{S}(\mathbf{D})$ is the equivalent compliance of damaged materials and then we have

$$
\mathbf{S}(\mathbf{D}+d \mathbf{D})=\mathbf{S}(\mathbf{D})+\frac{1}{2} d \lambda \cdot \mathbf{J}(\mathbf{D})
$$

In view of the conventional analysis $[3,4,12]$ for an isotropic damaged material, the effective compliance is proposed as

$$
\mathbf{S}(\mathfrak{D})=\mathfrak{D} \cdot \mathbf{S}(0)
$$

or

$$
\mathbf{S}(\mathfrak{D}+d \mathfrak{D})=\mathbf{S}(\mathfrak{D})+d \mathfrak{D} \cdot \mathbf{S}(0)
$$

where $\mathfrak{D}$ is a properly defined scalar damage variable [3] as in (2.15). Substitute (2.15) into (4.19) and express $\mathbf{J}$ as

$$
\mathbf{J}(\mathbf{D})=\mathbf{J}^{0}+\mathbf{J}^{\mathbf{1}}(\mathbf{D})=\mathbf{J}^{0}+D \cdot \mathbf{J}^{1}(\mathbf{I})
$$

where for the sake of simplicity, we establish from (4.6) that the components of $\mathbf{J}^{0}$ are

$$
\mathbf{J}^{0}=\left.\mathbf{J}(\mathbf{D})\right|_{\boldsymbol{D}=0}
$$

and

$$
\mathbf{J}^{1}(\mathbf{D})=\mathbf{J}(\mathbf{D})-\mathbf{J}^{0}
$$

The effective compliance presented in (4.20) contains a severe limitation as discussed in Section 2 in that there is no effect of Poisson's ratio on isotropic damage entities and the formulation is not therefore realistic. This is also elucidated by Chow and Wei [26] that the reduction of Poisson's ratio is a measurable quantity for aluminium alloys, even though the damage is isotropic. In view of this relevation, a damaged material, although it is isotropic in its virgin state, may be assumed to behave as an equivalent isotropic material when the damage variable is reduced to (2.15). Therefore, since $\mathbf{J}$ is considered as a material constant independent of damage entities or material deforming states, we can thus deduce from the above assumption that the coefficients of $\mathbf{J}$ in (4.6) may be readily given as

$$
\left.\begin{array}{ll}
a_{i}=\alpha, \quad(i=1,3,5) ; & a_{j}=\beta, \quad(j=2,4,6) \\
b_{i}=-\zeta / \alpha, \quad(i=1,4,7) ; & b_{i}=-\xi / 2 \beta, \quad(i=2,3,5,6,8,9) \\
c_{i}=-2(1+\zeta) / \alpha, \quad(i=1,4,7) ; & c_{i}=-(1+\xi) / 2 \beta, \quad(i=2,3,5,6,8,9) \\
e_{i}=d_{i}=0, \quad(i=1,3,5) & e_{i}=\eta \& d_{i}=v, \quad(i=2,4,6)
\end{array}\right\}
$$

where $\alpha, \beta, \zeta, \xi, v$ and $\eta$ are material constants which can be measured experimentally. 
In order to compare the above results with those evaluated from the conventional models, we simplify the discussion by confining the problem, to the damage principle coordinate system. The damage tensor shown in (2.13) may be reduced to

$$
\mathbf{D}=\operatorname{diag}\left(D_{11}, D_{22}, D_{33}\right)
$$

Then the conventional effective stress defined by the damage effect tensor $\mathbb{M}(\mathbb{D})$ and its associated damage variable $\mathbb{D}$, as defined in conventional models $[1,12,16]$ may be given by

$$
\tilde{\boldsymbol{\sigma}}=\mathbb{M}(\mathbb{D}): \boldsymbol{\sigma}
$$

Similarly, we define the effective damage stress for the proposed damage model as

$$
\tilde{\boldsymbol{\sigma}}^{d}=\mathbf{M}(\mathbf{D}): \boldsymbol{\sigma}^{d}
$$

for which the difference between $\mathbb{M}(\mathbb{D})$ and $\mathbf{M}(\mathbf{D})$ is to be identified. Without loss of generality, consider the following form of $F_{d}\left(\sigma^{d}, \mathrm{D}\right)$

$$
F_{d}\left(\boldsymbol{\sigma}^{d}, \mathbf{D}\right)=\boldsymbol{\sigma}^{T, d}: \mathbf{J}(\mathbf{D}): \boldsymbol{\sigma}^{d}=\boldsymbol{\sigma}^{T, d}: \mathbf{M}^{T}(\mathbf{D}): \mathbf{S}(0): \mathbf{M}(\mathbf{D}): \boldsymbol{\sigma}^{d}
$$

In addition, suppose that the current state of damage can be decomposed by

$$
\mathbf{D}=\sum_{1}^{n} \mathbf{d D}_{i} ; \quad \text { and } \quad \mathbf{D}_{i}=\sum_{1}^{i} d \mathbf{D}_{i}
$$

From (4.19)

$$
\begin{aligned}
& \mathbf{S}\left(d \mathbf{D}_{1}\right)=\mathbf{S}(0)+\frac{1}{2} d \lambda_{1} \cdot \mathbf{M}^{T}(0): \mathbf{S}(0): \mathbf{M}(0) \\
& \vdots \\
& \mathbf{S}\left(\mathbf{D}_{i+1}\right)=\mathbf{S}\left(\mathbf{D}_{i}\right)+\frac{1}{2} d \lambda_{i} \cdot \mathbf{M}^{T}\left(\mathbf{D}_{i}\right): \mathbf{S}(0): \mathbf{M}\left(\mathbf{D}_{i}\right) \\
& \vdots \\
& \mathbf{S}\left(\mathbf{D}_{n}\right)=\mathbf{S}\left(\mathbf{D}_{n-1}\right)+\frac{1}{2} d \lambda_{n-1} \cdot \mathbf{M}^{\mathbf{T}}\left(\mathbf{D}_{n-1}\right): \mathbf{S}(0): \mathbf{M}\left(\mathbf{D}_{n-1}\right)
\end{aligned}
$$

then the effective compliance may be given as

$$
\begin{aligned}
\mathbf{S}\left(\mathbf{D}_{n}\right)= & \mathbb{M}^{T}(\mathbb{D}): \mathbf{S}(0): \mathbb{M}(\mathbb{D})=\mathbf{S}(0)+\left[\frac{1}{2} d \lambda_{1} \cdot \mathbf{M}^{T}(0): \mathbf{S}(\mathbf{0}): \mathbf{M}(0)\right. \\
& +\cdots+\frac{1}{2} d \lambda_{i-1} \cdot \mathbf{M}^{T}\left(\mathbf{D}_{i-1}\right): \mathbf{S}(0): \mathbf{M}\left(\mathbf{D}_{i-1}\right)+\cdots+ \\
& \frac{1}{2} d \lambda_{n-1} \cdot \mathbf{M}^{T}\left(\mathbf{D}_{n-1}\right): \mathbf{S}(0): \mathbf{M}\left(\mathbf{D}_{n-1}\right) .
\end{aligned}
$$

It may thus be deduced from the above relation that

$$
\begin{aligned}
\mathbb{M}(\mathbb{D})= & \mathbf{I}+\frac{1}{2} d \lambda_{1} \cdot \mathbf{M}\left(\mathbf{D}_{1}\right)+\cdots+\frac{1}{2} d \lambda_{i-1} \cdot \mathbf{M}\left(\mathbf{D}_{i-1}\right)+ \\
& +\cdots+\frac{1}{2} d \lambda_{n-1} \cdot \mathbf{M}\left(\mathbf{D}_{n-1}\right) .
\end{aligned}
$$

A similar formulation has also been derived by Chow and Lu [27]. 
Take for example a conventional model of $\mathbb{M}(\mathbb{D})$ described in [12]. In the case of isotropic damage, $\mathbb{D}=\mathfrak{D} \cdot \operatorname{diag}(1,1,1)$. Then

$$
\begin{aligned}
\frac{1}{(1-\mathfrak{D})^{2}}= & 1+2 \mathfrak{D}+\cdots=1+2 \cdot\left\{\frac{1}{4} d \lambda_{1} \cdot\left(\alpha+\beta D_{1}\right)+\cdots+\right. \\
& \left.+\frac{1}{4} d \lambda_{n-1} \cdot\left(\alpha+\beta D_{n-1}\right)\right\}
\end{aligned}
$$

for which $\mathbf{M}(\mathbf{D})$ is defined as $\sqrt{\left(\alpha+\beta D_{1}\right)} \cdot \operatorname{diag}(1,1,1,1,1,1)$.

It is obvious that this conventional model $\mathbb{M}(\mathbb{D})[1,12,16]$ results in a first order approximation to (4.34) of the proposed model. However, in more general cases, the exact formulation of effective stiffness (or compliance) with damage should be made according to (4.19) as $\mathbf{M ( D )}$ would not be expressed by the above diagonal tensor and by the associated relation between $M(D)$ and $M(\mathbb{D})$ shown in (4.33).

\section{The elastoplastic coupled with anisotropic damage}

Once a loaded material undergoes plastic deformation, its mechanical properties may be degraded due to void stretching, wedge cracking, precipitate coarsening, increase in mobile dislocation density, formation of substructure, etc. The discussion for brittle anisotropic damage can also be applied to characterize elastoplastic damage by decomposing the increment of strain $d \varepsilon$ into $d \varepsilon^{e}+d \varepsilon^{p}$. Similarly, consider an infinitesimal deforming process with damage and plastic strain. The reference configurations $X_{t}$ at time $t$ are represented by $X_{t}\left(\sigma, \varepsilon^{e}+\varepsilon^{p}, \mathrm{D}\right)$, and $X_{t+d t}\left(\sigma+d \sigma, \varepsilon^{e}+\varepsilon^{p}+d \varepsilon, \mathbf{D}+d \mathbf{D}\right)$ at $t+d t$. The decomposed elastic and plastic strains are also coupled with damage effects as

$$
d \varepsilon=d \varepsilon^{e}+d \varepsilon^{p}
$$

Based on the incremental elastic deformation coupled with damage, the reference middle-state is proposed as $X_{t}^{\prime}\left(\sigma+d \sigma, \varepsilon^{e}+\varepsilon^{p}+d \hat{\varepsilon}^{p}+d \hat{\varepsilon}, \mathbf{D}\right)$ such that

$$
d \varepsilon^{e}=d \hat{\varepsilon}+d e^{d}
$$

where $d \varepsilon^{e}$ is the increment of current elastic strain tensor, $d \hat{\varepsilon}$ is defined as increment of partial elastic strain tensor at constant damage variable $\mathbf{D}$, and $d \varepsilon^{d}$, increment of damage strain tensor. Thus the increment of stress may be readily obtained as

$$
d \sigma=\mathbf{E}(\mathbf{D}, \omega): d \hat{\varepsilon}=\mathbf{E}(\mathbf{D}, \omega): d \varepsilon^{e}-\mathbf{E}(\mathbf{D}, \omega): \varepsilon^{d}
$$

Similarly, introducing the incremental elastic energy defined as

$$
d W^{e}=\boldsymbol{\varepsilon}^{e}: \mathbf{E}(\mathbf{D}, \omega): d \boldsymbol{\varepsilon}^{e}-\frac{1}{2} \varepsilon^{e}: \mathbf{E}(\mathbf{D}, \omega): d \varepsilon^{d}
$$

and

$$
\begin{aligned}
& d \Phi\left(\boldsymbol{\varepsilon}^{e}, \boldsymbol{\varepsilon}^{d}, \mathbf{D}, \omega, t\right)=d W^{e}+d\left(\Phi_{2}(\omega)\right)+d\left(\Phi_{1}(\alpha)\right) \\
& \quad=\varepsilon^{e}: \mathbf{E}(\mathbf{D}, \omega): d \varepsilon^{e}-\frac{1}{2} \varepsilon^{e}: \mathbf{E}(\mathbf{D}, \omega): d \varepsilon^{d}+d\left(\Phi_{2}(\omega)+d\left(\Phi_{1}(\alpha)\right)\right.
\end{aligned}
$$


and the Clausius-Duhem inequality becomes

$$
\sigma^{d}: d \varepsilon^{d}+\sigma: d \varepsilon^{p}+B_{1} \cdot d \omega+A \cdot \alpha \geqslant 0
$$

where $\alpha$ refers to accumulated plastic deformation and $A$ is its associated force.

On the other hand, if we assume that the plastic hardening potential function is not coupled with damage, $F_{p}(\sigma, A)$ can be readily presented as

$$
F_{p}(\sigma, A)=\sqrt{J_{2}}+F_{1}(A)
$$

In general, the plastic hardening function is coupled with damage which will be discussed later.

Experimental evidence reveals that there is no residual volume change for plastic deformation. The classical theory of plasticity assumes that the material plastic potential is only associated with its distortion energy, which is expressed via Levy-Mises flow theory

$$
F_{p}(\sigma)=\boldsymbol{\sigma}^{\prime T}: \sigma^{\prime}+F_{1}(A)
$$

and

$$
d \varepsilon^{p}=d \lambda \cdot \sigma^{\prime}
$$

Thus

$$
d v^{p}=d \varepsilon_{i i}^{p}=0
$$

where $\boldsymbol{\sigma}^{\prime}$ is the deviatoric tensor of $\boldsymbol{\sigma} ; d v^{p}$ represents residual volume change due to plastic deformation and the rate of plastic deformation $d \varepsilon^{p}$ is given by the assumption of convex and normality.

Similar to the classical plastic theory, the plastic potential function coupled with damage has been proposed by Chow and Wang [13] and generally presented as

$$
\begin{aligned}
F_{p}(\boldsymbol{\sigma}, \mathbf{D}) & =F_{p}(\tilde{\boldsymbol{\sigma}}, \mathbf{D})=\tilde{\boldsymbol{\sigma}}^{T}: \tilde{\boldsymbol{\sigma}}+F_{1}(A) \\
& =\tilde{\boldsymbol{\sigma}}^{T}: \mathbf{M}(\mathbf{D})^{T}: \mathbf{H}: \mathbf{M}(\mathbf{D}): \tilde{\boldsymbol{\sigma}}+F_{1}(A),
\end{aligned}
$$

where for isotropic materials

$$
H_{i j k l}=\frac{1}{2 v}\left[-\frac{1}{3} \delta_{i j} \cdot \delta_{k l}+\frac{1}{2}\left(\delta_{i k} \cdot \delta_{j l}+\delta_{i l} \cdot \delta_{j k}\right)\right]
$$

For (5.7), if we also assume that the material plastic deformation satisfies the convex and normality properties, the plastic deformation coupled with damage can also be presented as [13]

$$
\mathrm{d} \varepsilon^{p}=d \lambda_{p} \cdot \frac{\partial F}{\partial \boldsymbol{\sigma}}=d \lambda_{p} \cdot \tilde{\mathbf{H}}: \boldsymbol{\sigma}
$$

where

$$
\tilde{\mathbf{H}}=\mathbf{M}(\mathbf{D})^{T}: \mathbf{H}: \mathbf{M}(\mathbf{D})
$$


It is clear from the above that the condition of incompressibility is not satisfied or

$$
d v^{p}=d \varepsilon_{i i}^{p} \not \equiv 0
$$

In general, the damaged materials, as has been discussed previously, are considered to be orthotropic. For virgin anisotropic materials, Hill [28] first developed the Mises type of flow potential function as

$$
F_{p}(\boldsymbol{\sigma}, A)=\boldsymbol{\sigma}^{T}: \mathbf{H}: \boldsymbol{\sigma}+F_{1}(A)
$$

and assumed that

$$
\delta_{i j} \cdot H_{i j k l}=H_{i i k l}=0
$$

On the other hand, the plastic potential for isotropic materials, i.e. (5.7), is only considered to contain a strictly limited capacity of storing the distortion energy. A new plastic potential for anisotropic materials based on the theory of the eigen states in fourth order tensor has been proposed by Olszak et al. [29]. Its general form is also expressed as (5.14), but the tensor $\mathbf{H}$ is assumed to have the following properties:

(i) it has all the symmetry properties as elastic modulus $\mathbf{E}$ (or $\mathbf{S}$ ),

(ii) the spherical tensor is one of its eigen states with the corresponding eigen values to be zero,

(iii) each deviatoric eigen state of $\mathbf{S}$ (or $\mathbf{E}$ ) is also an eigen state of $\mathbf{H}$,

(iv) the corresponding flow function is a part of the total strain energy,

(v) it reduces to the form of $(6.1)$ in the isotropic case.

Then the tensor $\mathbf{H}$ is given as (see Appendix II)

$$
\mathbf{H}=\mathbf{S}-\frac{(\mathbf{I} \cdot \mathbf{S}) \otimes(\mathbf{S} \cdot \mathbf{I})}{\mathbf{I} \cdot \mathbf{S} \cdot \mathbf{I}}
$$

Thus, for a fixed quantity of damage variable, this concept of plastic flow to damaged materials may be extended to yield

$$
\mathbf{H}(\mathbf{D})=\mathbf{S}(\mathbf{D})-\frac{(\mathbf{I} \cdot \mathbf{S}(\mathbf{D})) \otimes(\mathbf{S}(\mathbf{D}) \cdot \mathbf{I})}{\mathbf{I} \cdot \mathbf{S}(\mathbf{D}) \cdot \mathbf{I}}
$$

or (using the Voigt notation)

$$
\begin{aligned}
& H_{i j k l}\left(D_{I J}\right)=S_{i j k l}\left(D_{I J}\right)-\frac{S_{m m i j}\left(D_{I J}\right) \cdot S_{n k k l}\left(D_{I J}\right)}{S_{q q r r}\left(D_{I J}\right)}(\mathrm{I}, \mathrm{J}=1,2,3) \\
& \quad \text { and } i, j, k, l, m, n=1,2,3 .
\end{aligned}
$$

Thus the plastic potential function coupled with damage variable is proposed in this paper as

$$
F_{p}(\sigma, \mathbf{D})=\sigma^{T}: \mathbf{H}(\mathbf{D}): \sigma+F_{1}(A)
$$


and

$$
d_{\varepsilon}^{p}=d \lambda_{p} \cdot \mathbf{H}(\mathbf{D}): \sigma
$$

where

$$
\left.\begin{array}{ll}
d \lambda_{p}=0 & F_{p}<0 \text { or } F_{p}=0 \text { and } \\
& \frac{\partial F_{p}}{\partial \boldsymbol{\sigma}}: d \boldsymbol{\sigma}+\frac{\partial F_{p}}{\partial \mathbf{D}}: d \mathbf{D}<=0 \\
d \lambda_{p}=\frac{\frac{\partial F_{p}}{\partial \boldsymbol{\sigma}}: d \boldsymbol{\sigma}+\frac{\partial F_{p}}{\partial \mathbf{D}}: d \mathbf{D}}{\left(F_{1}^{\prime}(A)\right)^{2} \cdot A^{\prime}(\alpha)} & \begin{array}{l}
F_{p}>=0 \text { and } \\
\frac{\partial F_{p}}{\partial \boldsymbol{\sigma}}: d \boldsymbol{\sigma}+\frac{\partial F_{p}}{\partial \mathbf{D}}: d \mathbf{D}>0
\end{array}
\end{array}\right\}
$$

and

$$
\delta_{i j} \cdot H_{i j k l}\left(D_{I J}\right)=H_{i i k l}\left(D_{I J}\right)=0 \quad(I, J=1,2,3)
$$

or

$$
d v^{p}=d \varepsilon_{i i}^{p}=0
$$

which indicates that the resident change of volume is vanished for the proposed elastoplastic model coupled with damage. On the other hand, the evolution law of damage can be derived similarly as brittle material, as discussed in Section 4.

\section{Conclusions}

The damage mechanics models currently available based on the macro-mechanics approach are primarily concerned with the formulation of the effective stiffness due to damage by introducing 'damage effect tensor' through the hypothesis of elastic energy equivalence. In comparison, the proposed model is focused on the preservation of symmetric properties caused by such microstructural changes as an alternative damage variable with the concept of volume average in a material element containing a cloud of microdefects. This approach enables the formulation of a simple second order damage tensor. The establishment of a constitutive equation with damage is thus derived by employing the increment of 'damage strain' $\varepsilon^{d}$ within the confine of an infinitesimal deforming process. The advantages of the proposed model are that it presents a clear physical representation and its resulting 'incremental form of constitutive equation' may be simply incorporated into the finite element analysis.

The proposed model assumes that the virgin material is isotropic and its damaged state is orthotropic. For most conventional metals, these two assumptions are considered reasonable and present a good approximation to characterize common engineering materials. However, for virgin orthotropic materials, the proposed model may be readily modified by re-formulating the J-tensor using the same irreducible integrity bases described in this paper. The irreducible 
integrity bases employed are in fact derived according to the virgin orthotropic materials, although all the derivations and formulae can also be obtained by using the irreducible integrity bases of virgin isotropic materials.

\section{Appendix I}

The integrity bases for tensor $\sigma^{d}$ and $\mathbf{D}$ are given by Spencer [26] under orthotropic transformation based on the first order approximation of damage $\mathbf{D}$ as

$$
\left.\begin{array}{l}
I_{1}=\sigma_{11}^{d}, \quad I_{2}=\sigma_{22}^{d}, \quad I_{3}=\sigma_{33}^{d}, \\
I_{4}=\left(\sigma_{23}^{d}\right)^{2}, \quad I_{5}=\left(\sigma_{13}^{d}\right)^{2}, \quad I_{6}=\left(\sigma_{12}^{d}\right)^{2}, \\
I_{7}=D_{11}, \quad I_{8}=D_{22}, \quad I_{9}=D_{33}, \\
I_{10}=D_{12}^{2}, \quad I_{11}=D_{23}^{2}, \quad I_{12}=D_{13}^{2}, \\
I_{13}=D_{23} \sigma_{23}^{d}, \quad I_{14}=D_{31} \sigma_{31}^{d}, \quad I_{15}=D_{12} \sigma_{12}^{d}, \\
I_{16}=D_{23} \sigma_{13}^{d} \sigma_{12}^{d}, \quad I_{17}=D_{31} \sigma_{12}^{d} \sigma_{23}^{d}, \quad I_{18}=D_{12} \sigma_{23}^{d} \sigma_{13}^{d} .
\end{array}\right\}
$$

where $D_{i j}$ are the components of tensor $\mathbf{D}$ which has been defined in (2.14). Then, the scalar function determined by $I_{k}(k=1-18)$ can be formulated as

$$
\hat{F}_{d}\left(\sigma_{d}, \mathbf{D}\right)=\hat{F}_{d}\left(I_{k}\right)=c_{k} \cdot I_{k}+e_{k l} \cdot I_{k} \cdot I_{l}
$$

where $c_{k}$, and $e_{k l}$ are material constants already discussed in Section 4.

\section{Appendix II}

Let the elastic compliance $\mathbf{S}$ be a symmetric fourth order tensor. If there exists a symmetric second order tensor a that satisfies

$$
\mathbf{S} \cdot \mathbf{a}=\lambda \cdot \mathbf{a}
$$

then we define the tensor a as the elastic eigen state of $\mathbf{S}$, and $\lambda$ is the modulus of the compliance. For stress tensor, it may form a six-dimensional space of second order symmetric $F_{2}^{s}$, resulting in six orthogonal eigen basis tensors

$$
\text { a } \quad I=1,2,-, 6
$$

and the corresponding eigen values are $\lambda_{I}$. Then

$$
\mathbf{S}=\left(\mathbf{S} \cdot \mathbf{a}_{1}\right) \otimes \mathbf{a}_{1}+\left(\mathbf{S} \cdot \mathbf{a}_{2}\right) \otimes \mathbf{a}_{2}+\cdots+\left(\mathbf{S} \cdot \mathbf{a}_{6}\right) \otimes \mathbf{a}_{6},
$$

or

$$
\mathbf{S}=\lambda_{1} \cdot \mathbf{a}_{1} \otimes \mathbf{a}_{1}+\lambda_{2} \cdot \mathbf{a}_{2} \otimes \mathbf{a}_{2}+\cdots+\lambda_{2} \cdot \mathbf{a}_{2} \otimes \mathbf{a}_{2} .
$$


Consider the properties of tensor $\mathbf{H}$ presented in Section 6. It can be easily verified that the expression of (6.11) satisfies all of these conditions. Let $\mathbf{b}$ be an eigen tensor of $\mathbf{H}$, thus

$$
\mathbf{H} \cdot \mathbf{b}=\mu \cdot \mathbf{b}
$$

Then we know that $\mathbf{b}$ is also in the space $F_{2}^{s}$, hence

$$
\mathbf{b}=b_{I} \cdot \mathbf{a}_{I}
$$

Substitute (A2.6) into (A2.5)

$$
b_{I} \cdot \mathbf{H} \cdot \mathbf{a}_{I}=\mu \cdot b_{I} \cdot \mathbf{a}_{I},
$$

and therefore the set of six linear homogeneous equations for $b_{I}$ is given as

$$
b_{I} \mathbf{a}_{J} \cdot \mathbf{H} \cdot \mathbf{a}_{I}=\mu \cdot b_{J} \quad(J=1,2-6)
$$

There are nontrivial solutions of the equation if the compatibility condition is satisfied

$$
\operatorname{det}\left(\mathbf{a}_{J} \cdot \mathbf{H} \cdot \mathbf{a}_{I}-\mu \cdot \delta_{I J}\right)=0 \quad(I, J=1,2,-, 6)
$$

Then we obtain the six order equation for eigen value $\mu$ as

$$
G^{6}=\mu^{6}+\alpha_{1} \mu^{5}+\alpha_{2} \mu^{4}+\alpha_{3} \mu^{3}+\alpha_{4} \mu^{2}+\alpha_{5} \mu+\alpha_{6}=0
$$

It can be verified that

$$
\alpha_{6}=0
$$

which agrees with the fact that the spherical tensor is an eigen state of $\mathbf{H}$ tensor with zero eigen value.

Although the properties satisfied by the tensor of $\mathbf{H}$ represent a special case, the plastic potential tensor $\mathbf{H}$ presented in (6.11) may be extended to general anisotropic materials.

\section{References}

1. S. Murakami, in Continuum Damage Mechanics: Theory and Applications, D. Krajcinovic and J. Lemaitre (eds.) (1987) 91-115.

2. L.M. Kachanov, Izvestiya Akademii Nauk SSR, Otd Tech Nauk, no. 8 (1958) 26-31.

3. J. Lemaitre, in Proceedings ICM 1, Kyoto, Japan (1971).

4. J. Lemaitre and J.L. Chaboche, in Proceedings of IUTAM Symposium on Mechanics of Visco-elastic Media and Bodies, Springer-Verlag, Gothenburg, Sweden (1974).

5. D. Krajcinovic, Applied Mechanics Review 37, no. 1 (1984) 1-6.

6. J. Lemaitre, Journal of Engineering Materials and Technology 107 (1985) 83-89.

7. S. Murakami, JSME International Journal 30, no 263 (1987) 701-710.

8. J.L. Chaboche, in 2nd International Seminar on Incl. Analysis and Life Prediction in High Temperature Environment (1979) 9-14. 
9. J.L. Chaboche, in Mechanical Behavior of Anisotropic Solids, J.P. Bocher (ed.) (1982) 737-760.

10. F. Sidoroff, in Symposium IUT AM, Physical Non-linearities in Structural Analysis, SENLTS Mai (1980).

11. L.M. Kachanov, Introduction to Continuum Damage Mechanics, 8 Nijhoff, The Netherlands (1986)

12. C.L. Chow and J. Wang, International Journal of Fracture 33 (1987) 3-16.

13. C.L. Chow and J. Wang, Engineering Fracture Mechanics 30, No. 5 (1988) 547-563.

14. Ibid, 32, no. 4 (1989) 601-612.

15. Ibid, 33, no. 2 (1989) 309-317.

16. C.L. Chow and T.J. Lu, Engineering Fracture Mechanics 34, no. 3 (1989) 679-701.

17. J.E. Masters and K.L. Reifsnider, Damage in Composite Materials, ASTM STP 775, K.L. Reifsnider (ed.) (1982) $40-62$.

18. A.A. Vakulenko and L.M. Kachanov, Mech. Tverdogo Tiela, no. 4 (1971) 159-166.

19. D. Krajcinovic and G.U. Fonseka, Journal of Applied Mechanics 48 (1981) 809-815.

20. R. Alreja, Proceedings, Royal Society London A399 (1985) 195-216.

21. D.H. Allen and C.E. Harris, International Journal of Solids and Structures 23, No. 9 (1987) 1301-1318.

22. H. Lee, K. Peng and J. Wang, Engineering Fracture Mechanics 21, no. 5 (1985) 1031-1054.

23. A.J.M. Spencer, in Continuum Physics Vol. 1, part III, A.C. Eringen (ed.) (1971) 240-355.

24. R. Talreja, in Yielding, Damage and Failure of Anisotropic Solids, EGF5, J.P. Boechler (ed.) Mechanical Engineering Publications, London (1989) 509-533.

25. J.W. Ju, International Journal of Solids and Structures 25, no. 7 (1989) 803-833.

26. C.L. Chow and Y. Wei, International Journal of Fracture 50 (1991) 301-316.

27. C.L. Chow and T.J. Lu, Theoretical and Applied Fracture Mechanics 14 (1990) 187-218.

28. R. Hill, The Mathematical Theory of Plasticity, Clarendon Press, Oxford (1950).

29. W. Olszak and J. Ostrowska-Maciejewska, Engineering Fracture Mechanics 21, no. 4 (1985) 625-632. 